\title{
CONTROL SYSTEM AND DESIGN OF THE MOTION MODULE OF A HETEROGENEOUS MODUlar MOBILE RobOT
}

\author{
Victor Andreev \& Valerii Kim
}
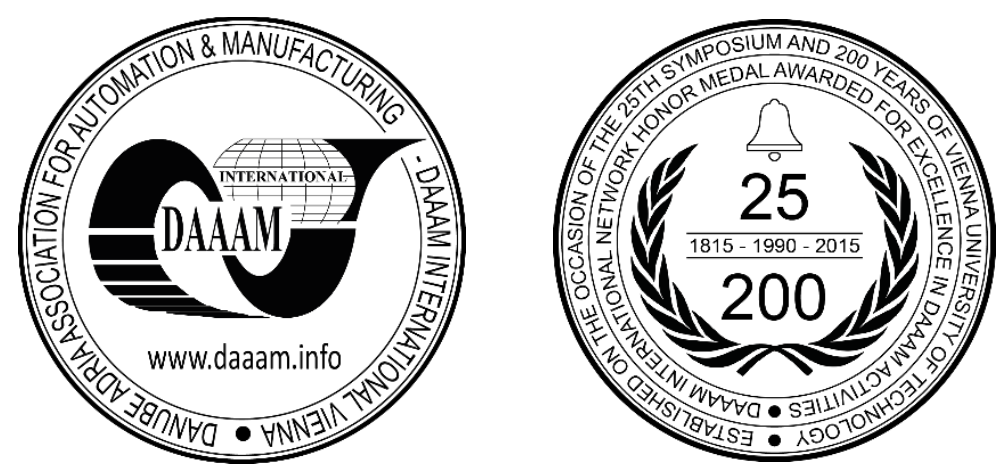

This Publication has to be referred as: Andreev, V[ictor] \& Kim, V[alerii] (2016). Control System and Design of the Motion Module of a Heterogeneous Modular Mobile Robot, Proceedings of the 27th DAAAM International Symposium, pp.0586-0594, B. Katalinic (Ed.), Published by DAAAM International, ISBN 978-3-902734-08-2, ISSN 1726-9679, Vienna, Austria

DOI: $10.2507 / 27$ th.daaam.proceedings.086

\begin{abstract}
We consider the modular principle for the construction of functional units of mobile robots. The main ideas of this concept and the general structure of the heterogeneous robot are presented. Basic functions of the robot are used to determine the minimum set of functional modules. For one of the simple configurations of the modular robot (the service mobile robot), we develop a motion module that is one of the main modules of the mobile robot. We present the design of this motion module and the result of a mathematical model of its control system. The control system combines the tactical level (for the construction of motion sub-targets and intermediate trajectories) and the executive level. The executive level of control system is based on kinematic and dynamic models of the modular robot. Working efficiency of the modular robot's model was tested on different motion tasks.
\end{abstract}

Keywords: mobile robot; modular robot; heterogeneous modular robot; mechanics of mobile robots; motion along trajectory; path planning; subordinate control loop; control system; servo drive; feedback control loop

\section{Introduction}

Normally, the design of robotic systems (RS) is accompanied by information about the purpose of the device. Accordingly, all the constructive and program decisions made during the design focus on the achievement of the specific purpose by the final device. Because of this, when the scope of application of the RS is changed, the designer has to revise in a varying degree the device construct ensuring its full compliance with the given purpose. One of the modern approaches to robotic design (and to machinery in general) is to divide the device structure into individual functional components. Each component is responsible for some part of the robot functionality. This unit or module normally has a simpler structure because it is responsible for a single function only. As one of many components of the robot, the module can be easily replaced by a similar or more advanced module. Then, there is no need to redesign the robot: it will suffice merely to replace it by particular functional units. Therefore, the modular robots can provide higher economic efficiency. 


\section{The modular principle in robotics}

The modular principle of mobile robot construction was introduced for the first time in the research of Yurevich E. I. [1]. Also the modular principle of design for industrial robots was implemented in work [2]. This principle became a separate research filed in robotics. One of the early work in this field is the CEBOT [3] robot described by Fukuda and Nakagawa. CEBOT consisted of similar modules-drives and its main purpose was to demonstrate that its configuration can be autonomously modified. Later, the CEBOT-type robots were said to be homogeneous robots, i.e., consisting of similar modules. In this paper, we consider another class of modular robots: heterogeneous modular robots. In heterogeneous robots, each module is responsible for a single function (or a pair of functions); accordingly, the modules of a single robot constructively may be very different. Heterogeneous robots have the advantage that they are more adapted to performing different tasks because the functionality of modules corresponds to the robot purpose and have tools to perform these tasks. However, at this stage, the majority of studies are devoted to homogeneous robots such as M-TRAN, ATRON, and SuperBot [4-6]. In heterogeneous robots, the reconfiguration task takes a back seat, and the versatility, flexibility, and interchangeability of modules becomes important. This fact was well reflected in Thor [7] and SMART [8] robots.

The purpose of this study is to develop a major functional unit of a modular mobile robot (MR) in the framework of the concept of modular system construction. We consider some specific features of this robot as well as what is meant by reconfiguration here.

The project concept is based on the following main principles of modular MR construction [9]:

1. Principle of constructive and functional independence of each module.

2. Principle of universal hardware and software communication interface and interaction between the modules.

3. Principle of distributed control.

4. Network principle of information-measuring and control system.

5. Each module is involved in the network structure with the help of special-purpose software (drivers).

The first principle means that each module should have a design and software that allow it to operate independently of other modules except power supply and most common instructions. This means that the design and software of a single module do not affect the operation of another module. Therefore each module of the modular MR is functional complete electronic or mechatronic device.

The second principle requires that the inter-module data communication be strictly standardized and independent of the type of the construction and control system (CS) of modules. This implies the following key feature of the given heterogeneous robot: the functional reconfigurability. This means that the inter-module interface should allow modules to execute their basic functions as a part of the robot that can be used to solving different kinds of tasks under different conditions of working environment. The final purpose of the robot determines only the design of modules and, in some cases, their control system, rather than the hardware and software interface of their interaction. For example, a service robot (Fig. 1a) can be converted into a track-mounted robot (Fig. 1b) relatively easily, without a significant change in the design of modules. The configuration of a go-anywhere modular MR (Fig. 1e) requires a change in the design and CS of only the motion module. In the case of transition to the underwater configuration (Fig. 1c), the designs of modules and their control systems should be significantly modified. In all the examples, the data interaction between modules will always be the same.
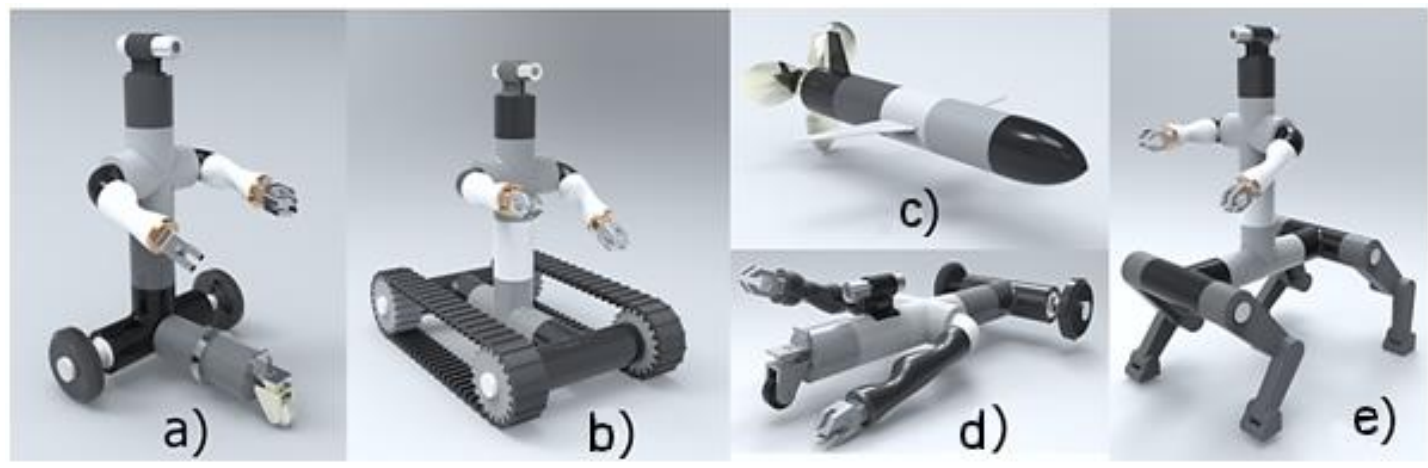

Fig. 1. Different configurations of the heterogeneous modular mobile robot.

The third principle determines the hierarchy of interaction between modules. The control of the operation of modules as a unified system is concerted by a special supervisory control system, which includes a control module as its core. This module forms objective functions for the remaining modules in the framework of the robot purpose. This is reached by dividing this task into common control instructions and their distribution between modules. Accordingly, this control instruction corresponds to the functionality of a given module. 
The fourth principle is based on the notion that each module is a unit of the local area network (LAN) [10]. As a result, the modular robot is a network structure with modules exchanging information with each other via standard network protocols (in this case, Ethernet).

The fifth principle, which is based on the network structure information-measuring and control system (IMCS) of the robot, serves as a major hardware and software interface between modules. The "principle of drivers" (its application for distributed control is considered in [11]) enables modules to be automatically involved in the common structure of the modular MR through special software (driver). In the future, not only the modules but also other devices manufactured by third parties can be included in the structure of modular MR.

Main aims of the study:

1. Develop a common structure of heterogeneous modular MR where each module is represented as an independent functional unit.

2. Determine the functionality of each module of the modular MR.

3. Develop the design and control system of the motion module as functional complete mechatronic device on the basis of the concept of modular MR and information about the robot purpose.

Based on the above-mentioned principles of the construction of heterogeneous modular MR, we have developed a common structure of the modular robot system (Fig. 2).

In this structure, each module corresponds to a certain function. The network control module (NCM) is the main control module responsible for supervisory control and distribution of common instructions between modules. The NCM interacts with each of the modules via the network hardware and software interface. Figure 2 shows that this system has four interfaces. As noted earlier, the design and CS of each module are determined by the robot purpose; however, the NCM structure is least dependent of this parameter.

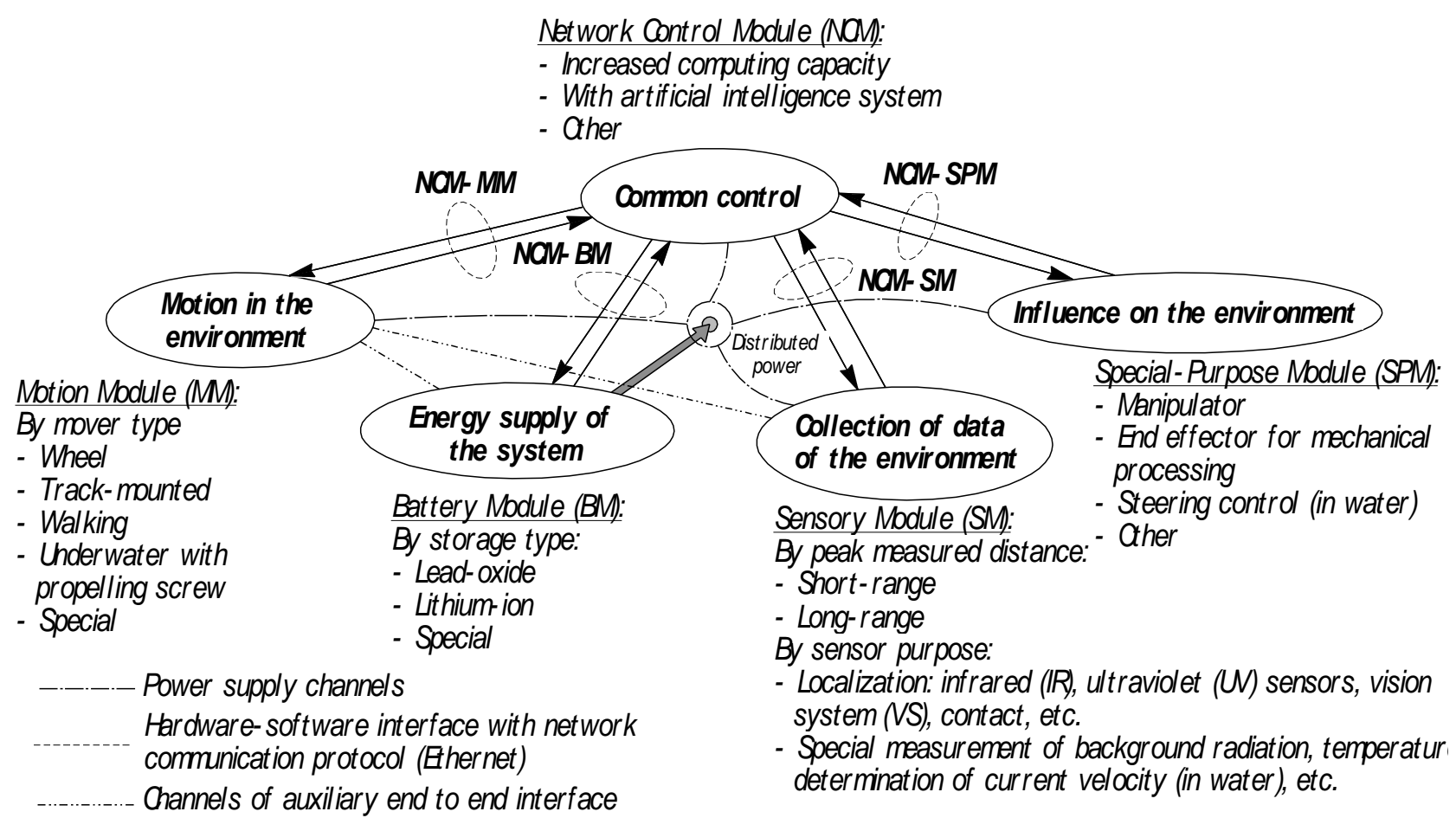

NOM-MM, NOM- BM, NCM- SM, NOM- SPM - interfaces of communication between corresponding moduls

Fig. 2. General structure of heterogeneous modular mobile robot.

In this project, the service modular MR is taken as the original configuration (see Fig. 1a) since this is the simplest assembly of the above-mentioned variants that is not subjected to special requirements.

Like other configurations, the service modular MR (according to the structure shown in Fig. 2) consists of the following modules (Fig. 3): 1 - Motion Module (MM), 2 - Battery Module (BM), 3 - Network Control Module (NCM), 4 - Special-Purpose Module (SPM), 5 - Sensory Module (SM). For this variant of the MR, the SPM is a module with manipulators. 


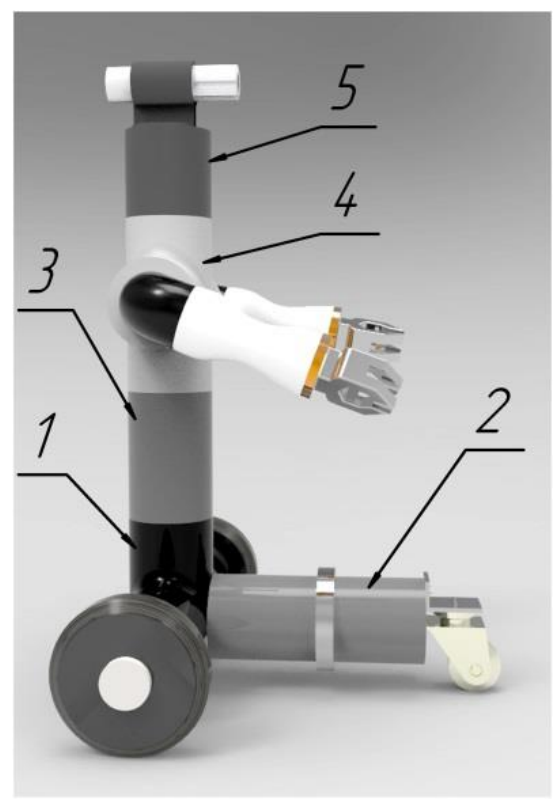

Fig. 3. Service modular mobile robot

\section{Design of the motion module}

The motion module (MM) is one of the main robot modules responsible for the function of motion in the environment (see Fig. 2). Since the service modular MR operates predominantly in indoor areas, the motion module should provide movement on flat surfaces.

The control system of the motion module combines both tactical and executive control levels. The tactical control level directly interacts with the NCM via the NCM-MM interface and receives from it necessary data to execute motion instructions: information about the coordinates and orientation of the robot in initial and final positions, data on the local map (if available), etc. In turn, the NCM receives actual data on the course of the instruction execution along the MM feedback chain.

The tactical level solves the problem of path planning and tracking along the trajectory. In this study, the tactical control level is implemented by the algorithm described in [12]. A similar approach is used for the mobile robot control [13]. Based on these studies, we developed a mathematical model of the CS of the tactical level using Simulink (MATLAB) to calculate the angular velocities of MM wheels aimed at its passing along a given trajectory.

The executive level of the CS consists of kinematic and dynamic models of the modular robot. Analysis of the motion module's control system is made along with considering battery module's dynamic parameters, because the battery module is presented in all configurations of the modular robot. The basis of the dynamic model are two servo drives with DC motors. Each servo drive is close looped with angular speed of the wheel of the motion module. Figure 4 shows the block diagram of the servo drive.

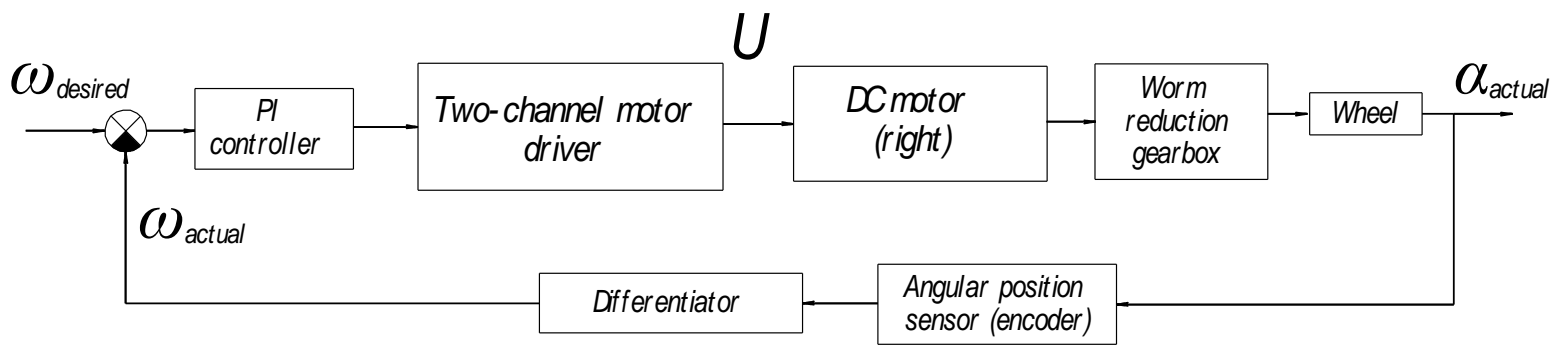

Fig. 4. Structural diagram of the servo drive

The servo drive's controller consists of the PI speed controller with the following transfer function:

$$
W_{P I}(s)=K_{P C} \frac{1+T_{P I} s}{T_{P I} S}
$$

where $T_{P I}$ - time constant of the PI speed controller, $K_{p c}-$ PI controller's gain coefficient. 
The PI controller is configured on a technical optimum [14]. In accord with this adjustment PI controller's time constant is equal to the time constant of the most inertial aperiodic link. Transfer function of an invariant part of the DC motor has aperiodic link with the electromechanical time constant $T_{M}$. It's well known that $T_{M}$ is much greater than electromagnetic time constant $T_{e}$ and motor driver's time constant $T_{P W M}$. Then PI controller's time constant is given by:

$$
T_{P I}=T_{M}=\frac{J_{\Sigma} R_{a}}{k_{m} k_{e}}
$$

where $J_{\Sigma}$ - summary reduced mass moment of inertia, $\mathrm{kg} \cdot \mathrm{m}^{2}, R_{a}$ - armature's winding's resistance, Ohm, $k_{m}-$ torque factor of the DC motor, $N \cdot m / A, k_{e}$ - coefficient of EMF of the DC motor, $V \cdot s / \mathrm{rad}$. Summary mass moment of inertia is given by (one wheel of considered drive rotates, another is fixed):

$$
J_{\Sigma}=\frac{J_{w}}{i^{2}}+J_{m}+M \cdot r^{2}+J_{z} \frac{r^{2}}{l^{2}}
$$

where $J_{w}$ - mass moment of inertia of the wheel relatively of it's rotation axis, $\mathrm{kg} \cdot \mathrm{m}^{2}, i-$ reduction ratio, $J_{m}-$ mass moment of a rotor's inertia, $\mathrm{kg} \cdot \mathrm{m}^{2}, M$ - mass of the motion module and the battery module, $\mathrm{kg}, r$ - wheel's radius, $m$, $J_{z}$ - mass moment of modular robot's inertia relatively of it's vertical rotation axis, $\mathrm{kg} \cdot \mathrm{m}^{2}, l$ - distance between wheels, $m$. Then PI controller time constant is:

$$
T_{P I}=\frac{R_{a}}{k_{m} k_{e}}\left(\frac{J_{w}}{i^{2}}+J_{m}+M \cdot r^{2}+J_{z} \frac{r^{2}}{l^{2}}\right) .
$$

PI controller's gain coefficient $K_{p c}$ proportional to Q factor of the speed control loop and estimated with consideration of the mass moment of inerta $J_{\Sigma}$.

Consideration of dynamic properties of the modular robot during evaluation of the PI controller's parameters allow to achieve acceptable quality of the servo drive control. Figure 5a shows transient speed process in servo drive and figure $5 \mathrm{~b}$ shows transient process with backlash in reduction gearbox (about $3^{\circ}$ ).

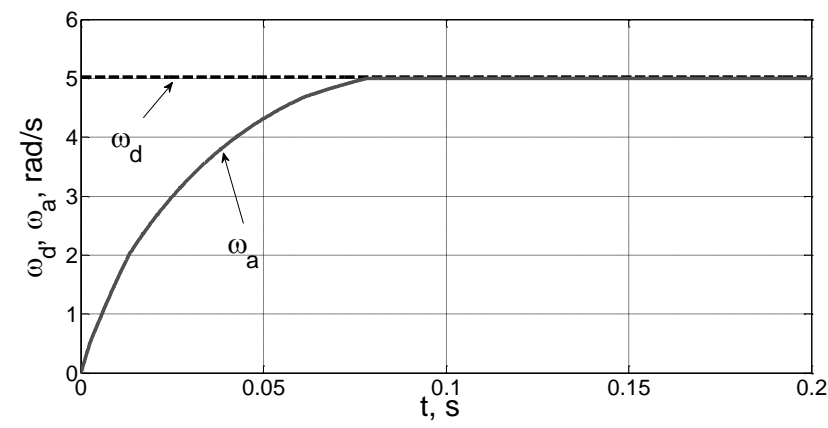

Fig. 5a. Transient speed process

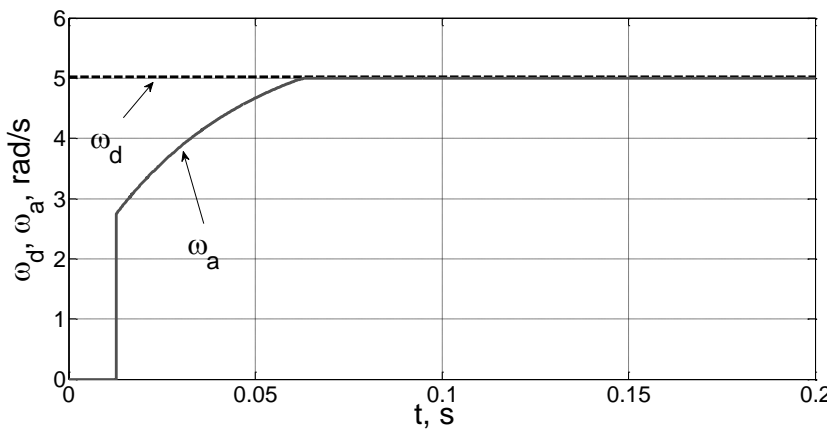

Fig. 5b. Transient speed process with gearbox' backlash

Analysis of graphs shows that overshoot is equal to zero, transient period is less than $0.07 \mathrm{~s}$ and stationary error is less than $0.4 \%$. Backlash in reduction gearbox causes speed's jump after short time delay. This can cause negative influence on motor driver and DC motor. It's necessary to use special correction algorithm for smooth speed control.

The computer model of the modular robot was developed. This model includes only executive level of the motion module's control system and sensor module. The sensor module is equipped with three laser rangefinders: one measures distance ahead of the robot, two others measure distance at rear sides of the robot. Figure 6 shows this model's structure developed in Simulink: 


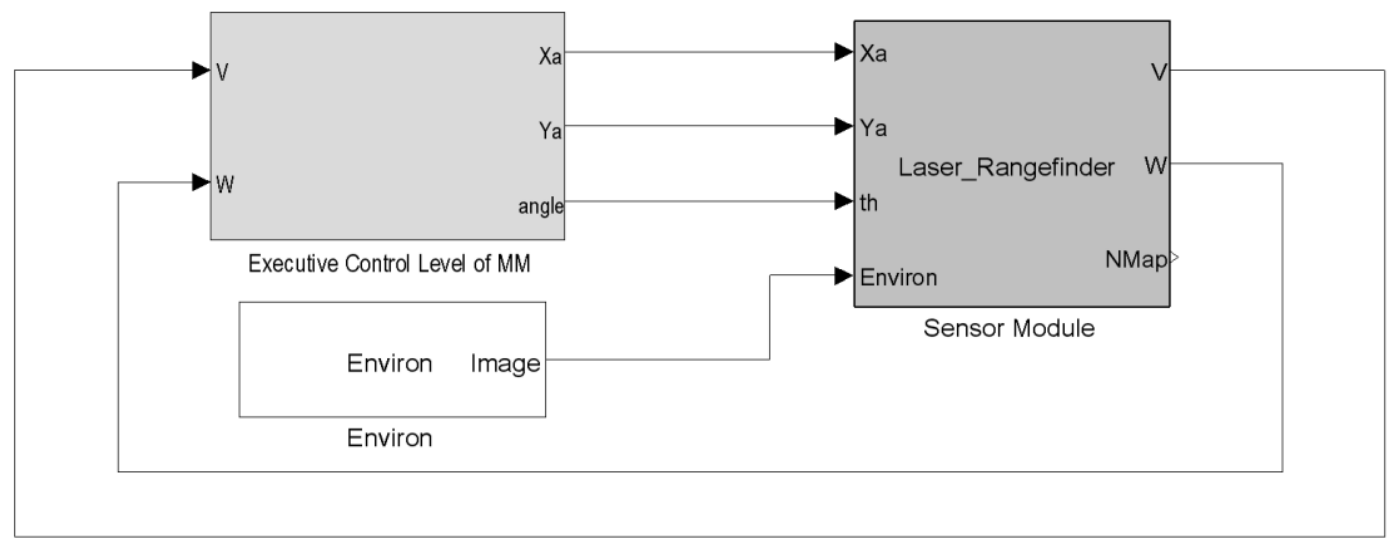

Fig. 6. Structure of modular robot's computer model

This modular robot's model is used for demonstration of the motion module capabilities to perform motion tasks in environment' model.

The executive control level of the motion module takes linear $V$ and angular $W$ velocities as input and actual position (coordinates $X a$ and $Y_{a}$ ) with orientation (angle) as output. The actual position and orientation of the robot is determined by the sensor module's SLAM algorithm (not considered in this work). The sensor module takes position and orientation of the modular robot and environment's model as input. Environment's model is represented here as image. Since in this model the executive control level of motion module can take as input only linear and angular velocities, the sensor module must executes its algorithms and gives $V$ and $W$ as output for feedback loop.

The modular robot's model was used for task of collision avoidance in room's model. For this task only one laser rangefinder in front of the modular robot was used in the sensor model. If the laser rangefinder detects an obstacle ahead of the robot on defined minimal distance then robot turn to left with angular speed $W$. Figure 7 shows the trajectory of the robot in the room with obstacles.



Fig. 7. The robot's trajectory in room with obstacles

From fig. 7 it's clear that the robot avoids obstacles located in front of the robot, but without laser rangefinders located at rear sides of the robot a collision with obstacles from right or left side may occur. This model experiment in only needed to demonstrate that the execution control level of the motion module can perform basic robot's movements.

The task of going through a labyrinth was considered. To perform this task the sensor module's computer model must have three laser rangefinders. Two laser rangefinders at rear sides of the modular robot directly used for the sensor module's algorithm. This algorithm is based on difference of distances between robot and side walls in labyrinth. If a distance to the right wall is greater than distance to the left wall then robot must turn on the right with angular speed $W$ and vice versa. Figure 8 shows the robot's trajectory in the labyrinth. 


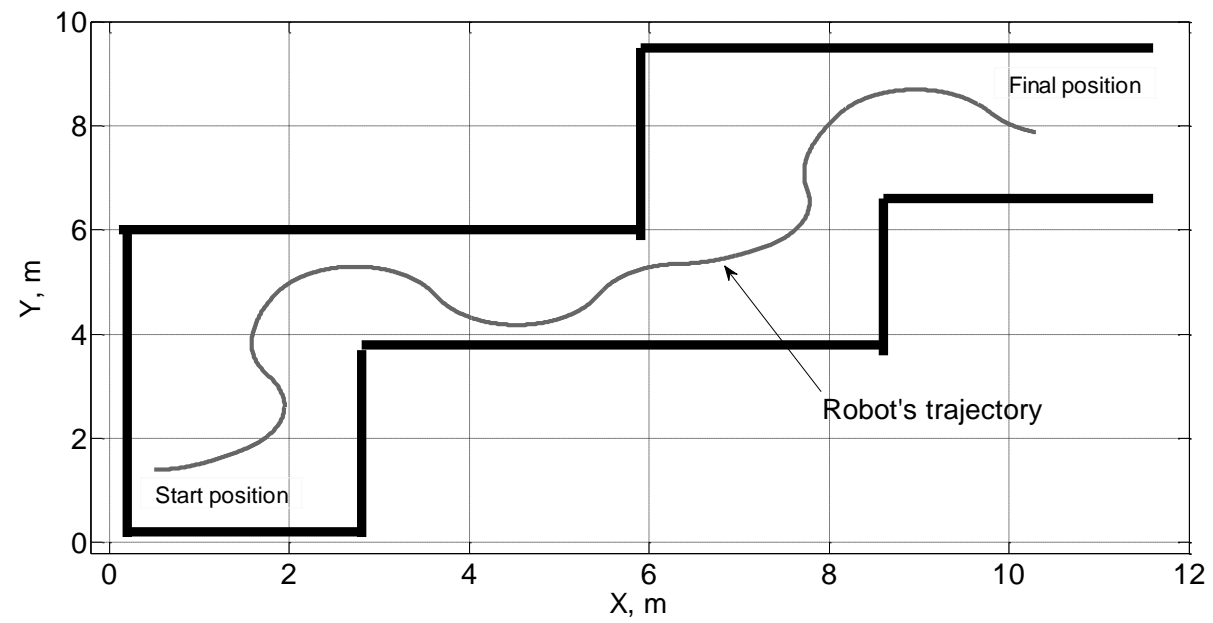

Fig. 8. Robot's trajectory in the labyrinth

From fig. 8 it is clear to see that the robot goes through labyrinth without collisions with walls. But the trajectory isn't optimal because the modular robot doesn't move straight where it would be more effective. This happens due to control algorithm that works without smooth correction of linear and angular velocities. Figure 9 shows graphs of laser rangefinder's measurements that were made during robot's motion through labyrinth.

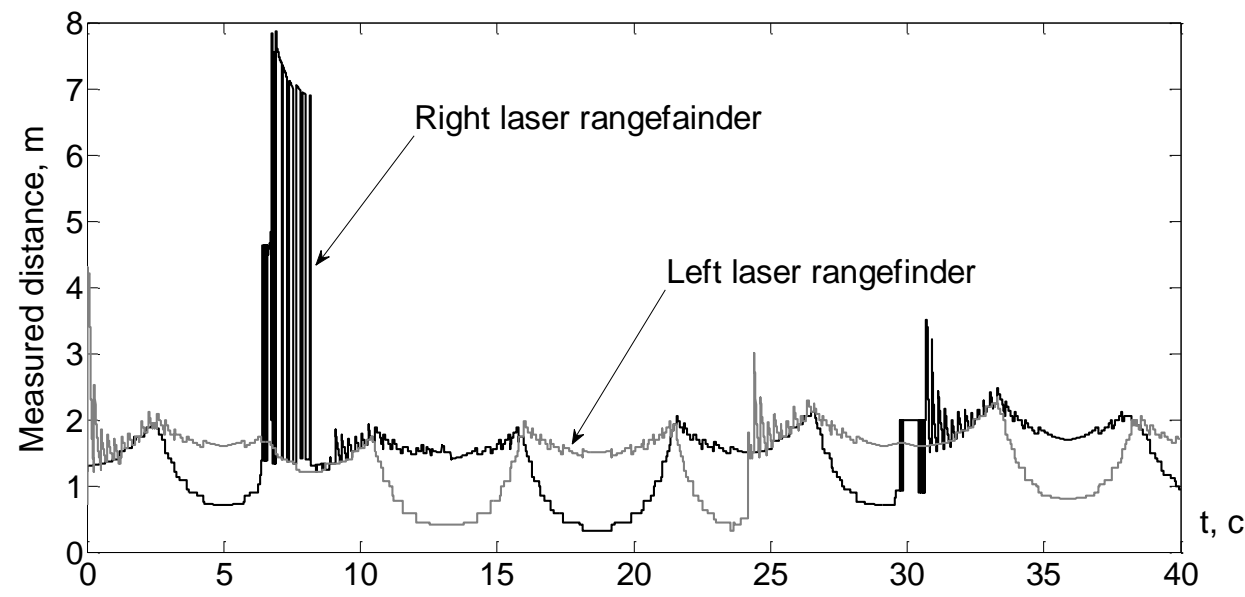

Fig. 9. Laser rangefinder's measurements

The jump in measured distance made by right laser rangefinder is due to detection one of the distant side walls in beginning of the motion.

As said before the tactical control level of motion module is implemented by the algorithm described in [12]. The computer model that was considered above was complemented by tactical level control block. Figure 10 shows tactical and executive control levels and sensor module.

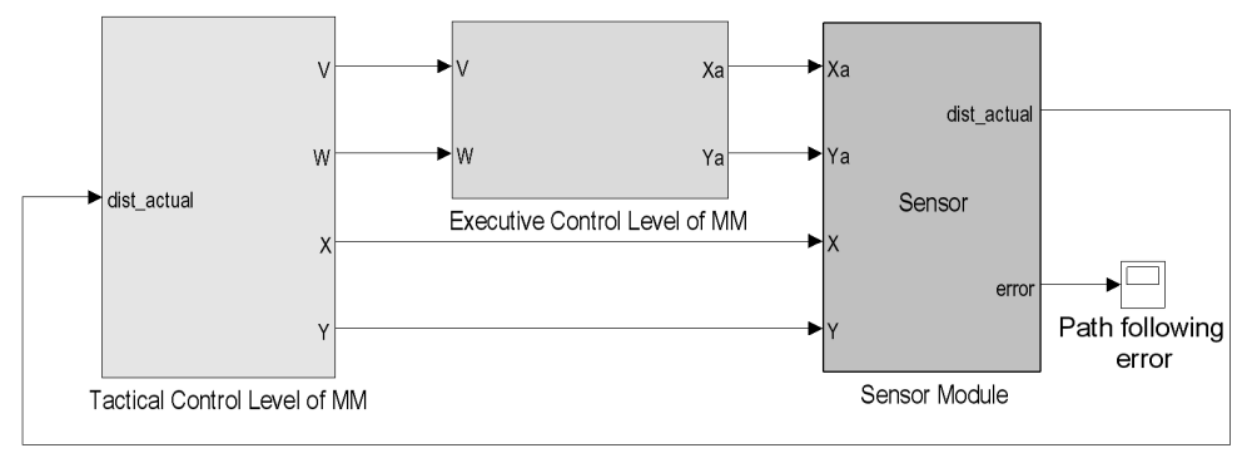

Fig. 10. Tactical and executive control levels of motion module with the sensor module 
Tactical control level evaluates intermediate aim positions and creates desirable trajectory of the modular robot. It takes actual distance of traversed path from the sensor module as input and desirable velocities $(V$ and $W)$ and robot's position as output. Executive control level of motion module takes desirable velocities as input and actual position as output. The sensor module takes actual and desirable positions as input and evaluates path following error and actual distance of traversed path. In this model we assume that the tactical control level already took coordinates of final position and desirable robot's orientation in this final position as input and created desirable trajectory. The modular mobile robot must move along a given trajectory with a least deviation from it. The modular robot can move from point to point along trajectory due to position control loop that organized by the sensor module. Figure 11 shows robot's actual and desirable mechanical trajectories after model simulation.

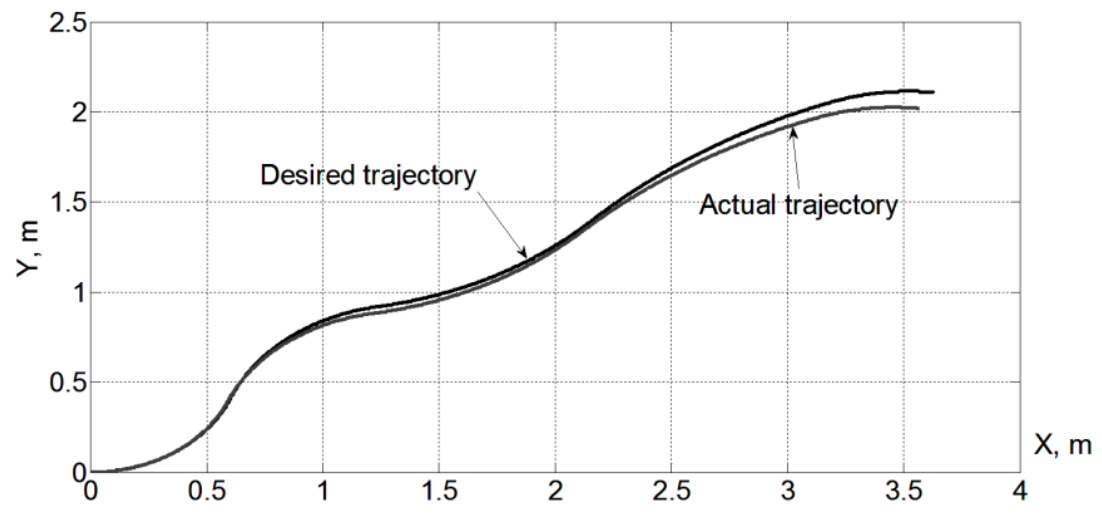

Fig. 11. Desired and actual modular robot's trajectories



Fig. 12. Motion module.

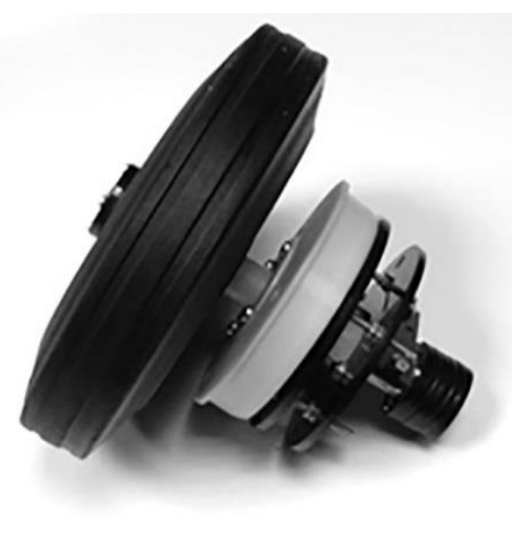

Fig. 13. Wheel-module.

The plot shows that the deviation of the actual motion from the desired one is sufficiently small (up to $35 \mathrm{~mm}$ ). We got similar results as in [12] but with greater path following error. The main source of a path following error is the inertia of the modular mobile robot and nonlinear elements in the dynamic robot's model. Development of correction algorithms for robot's position control loop is the objective for further research.Figure 12 shows the isometric section of the MM.

Let us consider some specific features of the MM design. The device consists of individual parts allowing us to relatively easily assemble the module (Fig. 12). The MM design contains two components connected to the drives and an electronic control unit. The MM drive consists of electric DC motor (1) and worm reduction gearbox (2). Gear (3) is connected to shaft (4) at the gearbox output through multilayer clutch (5). The shaft, clutch, frame unit (6), multilayer bearing sleeve (7), wheel (8), and encoder (9) constitute a single unit - wheel-submodule (Fig. 13.). The electronic control unit includes microcontroller-based hardware and software platform (10) and electric motor driver (11). Similar design decisions (usage of submodules for example) can be found in the modular robot Thor [7].

The described modular design of the mobile robot can be successfully used in Bionic Assembly System that presented in $[15,16]$. 


\section{Conclusions}

The main problem of this research was to create the functional complete motion module of the modular mobile robot. This problem was solved by designing special structure of the modular robot's system. At first stage we distributed basic functions of the robot between corresponding modules. Basic function of each module determines its hardware and software components. Principles of the modular mobile robot construction were considered during development of the motion module. Then control system of the module was designed: it's consists of both tactical and execution control levels. The control system receives data with simple structure (desirable position and orientation) and all complex calculations are performed by the motion module itself. At the same time the network control module must provide only actual data about desirable goal position and its software's structure is no matter for motion module. Working capacity of the control system's module was verified by computer modeling (the task of going through a labyrinth). In this model it's clear that interaction between the sensor module and the motion module is also realized by exchange of data with simple structure and with low dimension. In this paper we describe that the motion module's construction is constructively independent. This property is achieved by the simple mechanical interface and special construction arrangement of the module. Analysis of the control system and construction of the motion module allow concluding that this module is functional complete mechatronic device.

Development of the network control module and the sensor module, realization of natural experiments are objectives of further research. Design of the special-purpose module (a module with manipulators) is also objective of further research.

\section{Acknowledgments}

This work was supported by the Russian Foundation for Basic Research, project no. 16-07-00811a

\section{References}

[1]. A.V.Lopota, E.I.Yurevich, Stages and Developments of Robotic Systems Design Modular Principle, In St. Petersburg State Polytechnical University Journal, 1(164), St. Petersburg (Russia), 2013. - p.98-103.

[2]. Vorobiev, E.; Kozirev, Yu \& Tsarenko, V. (1988). Promishlennie roboti agregatno-modulnogo tipa (Industrial robots of unit-modular type) / Edited by E. P. Popov, Mashinostroenie, ISBN 5-217-00166-6, Moscow (Russia).

[3]. Fukuda T., S. Nakagawa, A dynamically reconfigurable robotic system (concept of a system and optimal configurations), In Proceedings of IECON '87: 1987 International Conference on Industrial Electronics, Control, and Instrumentation, 1987.

[4]. S. Murata, E. Yoshida, A. Kamimura, H. Kurokawa, K. Tomita, and S. Kokaji, M-TRAN: self-reconfigurable modular robotic system, IEEE/ASME Transactions on Mechatronics, 7(4):432-441, 2002.

[5]. E.H. Østergaard, K. Kassow, R. Beck, and H.H. Lund, Autonomous Robots, Design of the ATRON lattice-based self-reconfigurable robot, 21(2):165-183, 2006.

[6]. Salemi B., M. Moll, and W.-M. Shen, SUPERBOT: A deployable, multi-functional, and modular self-reconfigurable robotic system, In Proceedings, IEEE/RSJ Intl. Conf. on Intelligent Robots and Systems, pages 3636-3641, Beijing, China, 2006.

[7]. Lyder A.H., Stoy K., Mendoza-Garcia R.-F., Larsen J.C., and Hermansen P., On sub-modularization and morphological heterogeneity in modular robotics, In Sukhan Lee, Hyungsuck Cho, Kwang-Joon Yoon, and Jangmyung Lee (Eds.), Intelligent Autonomous Systems 12, volume 193 of Advances in Intelligent Systems and Computing, pp. 649-661. Springer Berlin Heidelberg, 2013.

[8]. J. Baca, M. Ferre, and R. Aracil, A heterogeneous modular robotic design for fast response to a diversity of tasks, Robotics and Autonomous Systems, vol. 60, no. 4, pp. 522-531, 2012.

[9]. Andreev, V[ictor], \& Poduraev, Y[ury] (2016). Network-based design of heterogeneous modular mobile robotic systems, Proceedings of the 27th DAAAM International Symposium, pp.xxxx-xxxx, B. Katalinic (Ed.), Published by DAAAM International, ISBN 978-3-902734-08-2, ISSN 1726-9679, Vienna, Austria DOI: 10.2507/27th.daaam.proceedings.xxx.

[10]. Pryanichnikov V.E., Andreev V.P., The Application of Network Technologies to Constructing Group Controlled Systems with Machine Vision for Mobile Robots, In Annals of DAAAM for 2012\& Proceedings of the 23th international DAAAM Symposium "Intelligent Manufacturing \& Automation" 24-27th October 2012 Zadar, Croatia, ISSN 2304-1382, 2012. - V.23, No.1. - pp.1167 - 1174.

[11]. Andreev V.P., Kirsanov K.B., Poduraev Yu. V., Geographically distributed “multi-operator" control of robotized systems using network technologies, In Proceedings of the International Scientific and Technical Conference "Extreme Robotics". - St.Petersburg: "Politechnika-service", 2015. - P.67-71.

[12]. Gulati S., Kuipers B., High performance control for graceful motion of an intelligent wheelchair, In Proc. 2008 IEEE Int. Conf. on Robotics and Automation (ICRA, May 2008), pp. 3932-3938.

[13]. Davydov O.I., Platonov A.K., A control algorithm for a differential drive of RB-2 mobile robot, Preprint Keldysh Institute of Applied Mathematics, RAS. Moscow. 2015. No. 25. - pp.4 - 16.

[14]. Kluchev V. (2001) Teoriya electroprivoda (Theory of electric servo drive), Energoatomizdat, ISBN 5-283-00642-5, Moscow (Russia).

[15]. Katalinic B., Kukushkin I., Pryanichnikov V., Haskovic D. Cloud Communication Concept for Bionic Assembly System. Procedia Engineering, 1877-7058, 69 (2014) (2014), pp. 1562-1568 doi:10.1016/j.proeng.2014.03.156.

[16]. Katalinic B., Kukushkin I., \& Haskovic D. Bionic Assembly System Cloud: Functions, Information Flow and Behavior. In 9th International Conference of DAAAM Baltic, Industrial Engineering, ISBN 978-9949-23-620-6, ISSN 2346-6138. pp. 103-108, ed. T.Otto, Tallinn, Estonia, 2014. 